\title{
A Medium-Chain Fatty Acid, Capric Acid, Inhibits RANKL-Induced Osteoclast Differentiation via the Suppression of NF-kB Signaling and Blocks Cytoskeletal Organization and Survival in Mature Osteoclasts
}

\author{
Hyun-Ju Kim ${ }^{1,2, *}$, Hye-Jin Yoon ${ }^{2}$, Shin-Yoon $\mathrm{Kim}^{3}$, and Young-Ran Yoon ${ }^{1,2, *}$
}

Fatty acids, important components of a normal diet, have been reported to play a role in bone metabolism. Osteoclasts are bone-resorbing cells that are responsible for many bone-destructive diseases such as osteoporosis. In this study, we investigated the impact of a medium-chain fatty acid, capric acid, on the osteoclast differentiation, function, and survival induced by receptor activator of NF-kB ligand (RANKL) and macrophage colony-stimulating factor (MCSF). Capric acid inhibited RANKL-mediated osteoclastogenesis in bone marrow-derived macrophages and suppressed RANKL-induced IKB $\alpha$ phosphorylation, p65 nuclear translocation, and NF-kB transcriptional activity. Capric acid further blocked the RANKL-stimulated activation of ERK without affecting JNK or p38. The induction of NFATc1 in response to RANKL was also attenuated by capric acid. In addition, capric acid abrogated M-CSF and RANKLmediated cytoskeleton reorganization, which is crucial for the efficient bone resorption of osteoclasts. Capric acid also increased apoptosis in mature osteoclasts through the induction of Bim expression and the suppression of ERK activation by M-CSF. Together, our results reveal that capric acid has inhibitory effects on osteoclast development. We therefore suggest that capric acid may have potential therapeutic implications for the treatment of bone resorption-associated disorders.

\footnotetext{
1Department of Molecular Medicine, Cell and Matrix Research Institute, School of Medicine, Kyungpook National University, Daegu 700-422, Korea, ${ }^{2}$ Clinical Trial Center, Kyungpook National University Hospital, Daegu 700-721, Korea, ${ }^{3}$ Department of Orthopedic Surgery, School of Medicine, Kyungpook National University, Daegu 700-422, Korea

*Correspondence: biohjk@ hanmail.net (HJK); yry@knu.ac.kr (YRY)
}

Received 5 June, 2014; revised 2 July, 2014; accepted 7 July, 2014; published online 18 August, 2014

Keywords: apoptosis, capric acid, cytoskeletal organization, NFATc1, $\mathrm{NF}-\mathrm{kB}$, osteoclast

\section{INTRODUCTION}

Osteoclasts are multinucleated cells that play a central role in adult skeletal homeostasis (Boyle et al., 2003; Martin, 2014; Teitelbaum and Ross, 2003). These bone-resorbing polykaryons are generated from the hematopoietic progenitor cells of the monocyte/macrophage lineage through multiple processes that include the fusion and differentiation of precursor cells and the activation of mature osteoclasts. To perform bone resorption, mature osteoclasts attach to bone surfaces and undergo cytoskeletal reorganization, forming actin rings at the sealing zone (Vaananen et al., 2000). Integrin, specifically $\alpha v \beta 3$, plays an essential role in the organization of actin cytoskeleton (Faccio et al., 2003; McHugh et al., 2000). Two key cytokines, macrophage colony-stimulating factor (M-CSF) and receptor activator of nuclear factor kappa B (NF-kB) ligand (RANKL), support osteoclast differentiation, function, and survival (Novack and Teitelbaum, 2008; Teitelbaum, 2007; Teitelbaum and Ross, 2003).

RANKL, a TNF family member, plays an essential role in osteoclast differentiation. The binding of RANKL to RANK activates $\mathrm{NF}-\kappa \mathrm{B}$ and mitogen-activated protein kinases (MAPKs), including c-Jun $\mathrm{N}$-terminal kinase (JNK), extracellular signal-regulated kinase (ERK), and p38, by recruiting TNF receptor-associated factor 6 (TRAF6) (Boyle et al., 2003). Previous studies have established that NF-kB activation is crucial for osteoclast differentiation (Franzoso et al., 1997; lotsova et al., 1997). The activation of NF-KB induces the expression of nuclear factor of activated T cells cytoplasmic 1 (NFATc1), which is a master transcription factor in osteoclastogenesis. NFATc1 can regulate the transcription of osteoclast-related markers, including tartrateresistant acid phosphatase (TRAP), cathepsin K, and MMP-9, during osteoclast differentiation (Asagiri and Takayanagi, 2007; Nakashima and Takayanagi, 2011).

Capric acid is a medium-chain fatty acid that occurs naturally in coconut oil. It is also found in the milk and fats of some mammals. As capric acid possesses strong antiviral, antibacterial, and antifungal properties, it is used as an antimicrobial pesticide. Fatty acids play an important role in the control of inflammatory diseases including obesity and diabetes. Interestingly, fatty acids have also been reported to modulate bone metabolism and 
bone mass (Cornish et al., 2008; Rahman et al., 2008; Sun et al., 2003). Although a recent study showed that several milk fractions and their hydrolysates suppressed osteoclast formation (Cornish et al., 2008), the molecular mechanisms of the medium-chain fatty acid, capric acid on RANKL-induced osteoclastogenesis are still poorly understood. In addition, the effect of capric acid on osteoclast cytoskeleton and survival has yet to be revealed.

In the present study, we demonstrate that capric acid suppresses osteoclast differentiation in bone marrow-derived macrophages (BMMs) through the suppression of RANKLstimulated signaling pathways, including NF- $\kappa B$ and ERK, as well as the induction of NFATc1. We also demonstrate that capric acid blocks cytoskeletal organization mediated by RANKL and M-CSF and accelerates the apoptosis of mature osteoclasts by regulating Bim expression and ERK activation.

\section{MATERIALS AND METHODS}

\section{Ethics statement}

All experimental procedures were performed in strict accordance with the appropriate institutional guidelines for animal research. The protocol was approved by the Committee on the Ethics of Animal Experiments of the Kyungpook National University.

\section{Reagents and antibodies}

Capric acid was purchased from Sigma (USA). RANKL and MCSF were obtained from R\&D Systems (USA). Antibodies against phospho-IKB $\alpha$, phospho-JNK, phospho-p38, phosphoERK, I $\mathrm{kB} \alpha$, JNK, p38, ERK, phospho-c-Jun, and nucleophosmin were purchased from Cell Signaling Technology (USA). Anti-Bim antibody was obtained from BD Biosciences (USA). Anti-p65 antibody was obtained from Santa Cruz Biotechnology (USA). Anti-NFATc1 antibody was purchased from BD Pharmingen (USA).

\section{Macrophage isolation and osteoclast culture}

Bone marrow was extracted from the femora and tibia of 6-8 week-old male C57/BL6 mice and cultured in $\alpha$-minimal essential medium (MEM) containing 10\% FBS and M-CSF. On day 3 of culture, the cells, selected for as a pure population of macrophages, were lifted and re-plated at $5 \times 10^{3}$ cells/well in a 96-well plate in $\alpha$-MEM containing $10 \%$ FBS with M-CSF $(10 \mathrm{ng} / \mathrm{ml})$ and RANKL $(20 \mathrm{ng} / \mathrm{ml})$. After 4 days, the cells were fixed in $4 \%$ paraformaldehyde for $20 \mathrm{~min}$ and then stained with tartrate-resistant acid phosphatase (TRAP) staining solution $(0.1 \mathrm{mg} / \mathrm{ml}$ naphthol AS-MX phosphate and $0.3 \mathrm{mg} / \mathrm{ml}$ Fast Red Violet LB stain).

\section{Cell viability assay}

Cell viability was determined using a colorimetric 3-(4,5dimethylthiazol-2-yl)-5-(3-carboxymethoxyphenyl)-2-(4-sulfophenyl)$2 \mathrm{H}$-tetrazolium (MTS) assay. BMMs were treated with various concentrations of capric acid for 3 days in the presence of M-CSF (30 $\mathrm{ng} / \mathrm{ml}$ ) in 96-well plates. Then, a medium containing $20 \mu$ of CellTiter 96 Aqueous One Solution (Promega, USA) was added. After $4 \mathrm{~h}$ of incubation, the absorbance was measured at $490 \mathrm{~nm}$ using a microplate reader.

\section{Real-time PCR}

Real-time PCR was performed as described previously (Kim et al., 2012). The following primers were used: NFATc1, 5'-ACCACCTTTCCGCAACCA-3' and 5'-TTCCGTTTCCCGTTGCA-3'; TRAP, 5'-TCCCCAATGCCCCATTC-3' and 5'-CGGTTCTGGCGATCTCTTTG-3'; cathepsin K, 5'-GGCTGTGGAGGCGGCTAT-3' and 5'-AGAGTCAATGCCTCCGTTCTG-3'; MMP-9, 5'-
AAAGACCTGAAAACCTCCAACCT-3' and 5'-GCCCGGGTGTAA CCA TAGC-3'; $\beta 3$ integrin, 5'-CGCATCCCATTTGCTAGTGTT-3' and 5'-CTGCCAGCCTTCCATCCA-3'.

\section{Western blotting}

Cells were harvested after washing with ice-cold PBS and then lysed in extraction buffer containing $50 \mathrm{mM}$ Tris- $\mathrm{HCl}(\mathrm{pH} 7.4)$, $150 \mathrm{mM} \mathrm{NaCl}, 1 \mathrm{mM}$ EDTA, $1 \%$ Nonidet P-40, and protease and phosphatase inhibitors for $30 \mathrm{~min}$ on ice. Protein concentrations of the cell lysates were determined using a bicinchoninic acid kit (Pierce, USA). Equal amounts of protein were resolved by SDS-PAGE and transferred to a polyvinylidene difluoride membrane (Millipore, USA). The membrane was probed with specific antibodies, and immuno-reactivity was detected using enhanced chemiluminescence reagents (ECL-plus, Amersham, USA).

Nuclear protein extraction

Cells were lysed with hypotonic buffer (10 mM Hepes, $1.5 \mathrm{mM}$ $\mathrm{MgCl}_{2}, 1 \mathrm{mM} \mathrm{KCl}, 1 \mathrm{mM} \mathrm{DTT}$, and protease and phosphatase inhibitors), followed by the addition of $0.1 \%$ Nonidet P-40. After centrifugation, the nuclear pellets were suspended in high-salt buffer (hypotonic buffer plus $400 \mathrm{mM} \mathrm{NaCl}$ ). Protein concentrations were determined, and immunoblotting was performed.

\section{Luciferase assay}

RAW264.7 cells were plated at a density of $3 \times 10^{5}$ cells/well in a 24-well plate. The next day, the cells were transfected with an NF-кB luciferase reporter construct. The transfected cells were incubated with increasing doses of capric acid for $24 \mathrm{~h}$ and then stimulated with RANKL $(200 \mathrm{ng} / \mathrm{ml})$ for $24 \mathrm{~h}$. The cells were lysed in Reporter Lysis Buffer (Promega), and the luciferase activity was measured using a luminometer.

\section{Apoptosis assay}

Osteoclasts derived from BMMs were cultured with vehicle or capric acid for $24 \mathrm{~h}$. An apoptosis assay was conducted using the Cell Death Detection ELISA Plus kit (Roche Applied Science, Switzerland), which detects cytoplasmic histone-associated DNA fragmentation. The absorbance at $405 \mathrm{~nm}$ was measured using a Bio-Rad microplate reader.

\section{Actin ring re-formation assay}

Mature osteoclasts generated on bone slices (IDS Nordic Bioscience, Denmark) were washed twice with cold cytokine-free medium followed by incubation at $37^{\circ} \mathrm{C}$ for $20 \mathrm{~min}$. The washed cells were then incubated with or without capric acid $(400 \mu \mathrm{M})$ in the presence of RANKL $(20 \mathrm{ng} / \mathrm{ml})$ or M-CSF $(50 \mathrm{ng} / \mathrm{ml})$ for $3 \mathrm{~h}$. The bone slices were fixed with $4 \%$ paraformaldehyde in phosphate-buffered saline for $20 \mathrm{~min}$. F-actin was stained with Alexa Fluor 488-phalloidin (Invitrogen).

\section{Statistical analysis}

All experiments were performed in triplicate. The data are presented as the means \pm S.D. Statistical significance was determined using a two-tailed Student's $t$-test.

\section{RESULTS}

\section{Capric acid inhibits RANKL-induced osteoclast} differentiation

To examine the effect of capric acid on RANKL-induced osteoc lastogenesis, BMMs were cultured with various concentrations of capric acid in the presence of RANKL and M-CSF for 4 days. The addition of capric acid inhibited the formation of TRAP- 


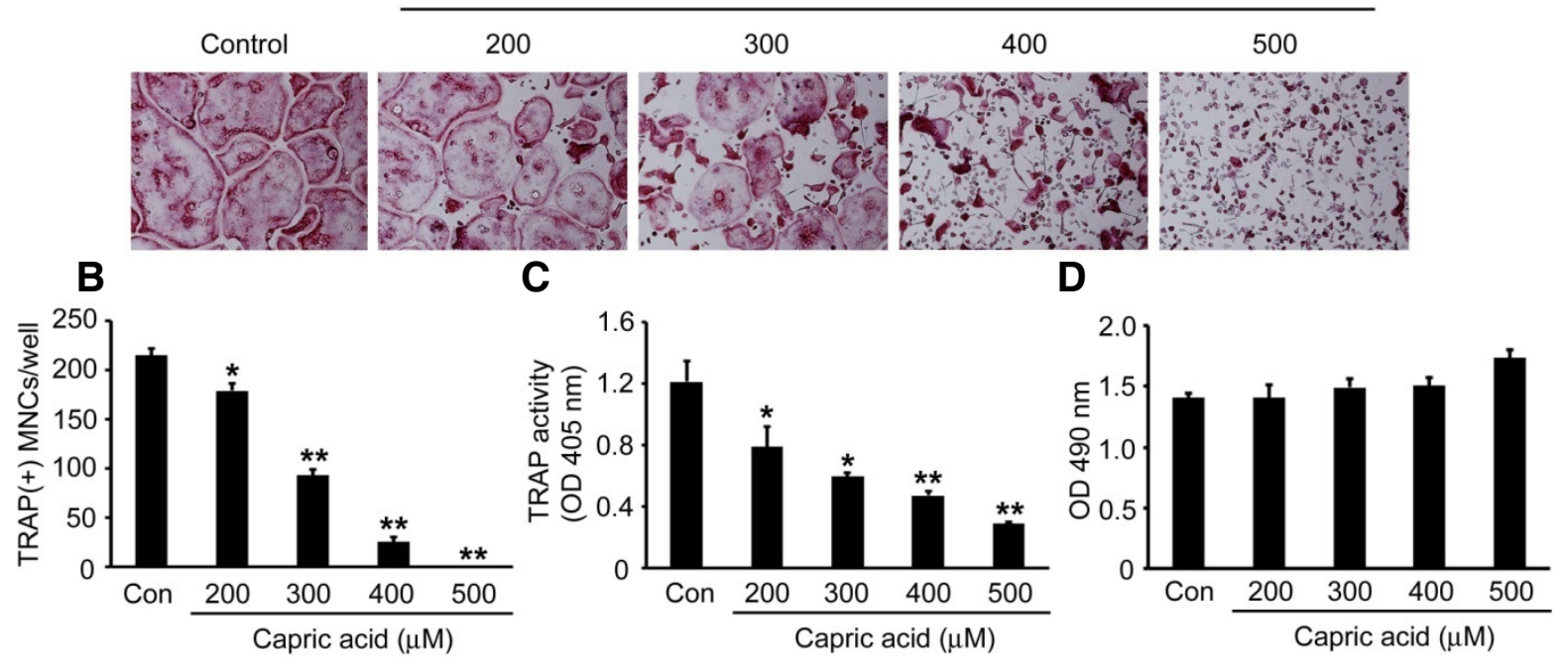

Fig. 1. Effect of capric acid on RANKL-induced osteoclast differentiation. BMMs were cultured with M-CSF (10 ng/ml) and RANKL (20 ng/ml) for 4 days at the indicated concentration of capric acid. (A) The cells were fixed and subjected to TRAP staining. (B) The number of TRAP-positive multinucleated cells (MNCs) generated in (A) was counted. (C) TRAP activity in the cell culture supernatant generated in (A) was measured by ELISA. The data presented in (B) and (C) are expressed as the means $\pm \mathrm{SD}$. ${ }^{*} P<0.05$, ${ }^{\star *} P<0.001$ versus vehicle-treated control (Con). (D) BMMs were cultured with M-CSF $(30 \mathrm{ng} / \mathrm{ml})$ for 3 days together with the indicated dose of capric acid. Cell viability was measured by an MTS assay, as described in "Materials and Methods".

positive multinuclear osteoclasts in a dose-dependent manner (Figs. 1A and 1B). Reflecting the decreased number of TRAPexpressing osteoclasts, TRAP activity measured in the culture supernatant was also reduced in the capric acid-treated cells (Fig. 1C). Using an MTS assay, we also observed that the addition of capric acid did not affect the viability of osteoclast precursors (BMMs) (Fig. 1D).

Because capric acid inhibited osteoclast formation, we next investigated whether capric acid could affect the expression of genes that play important roles in osteoclast differentiation. RANKL exposure in control cells induced the mRNA expression of NFATc1, which is a key regulator of osteoclastogenesis (Fig. $2 A)$. The addition of capric acid significantly attenuated the mRNA level of NFATc1 (Fig. 2A), and the suppressive effect of capric acid on NFATc1 expression was further confirmed by immunoblotting (Fig. 2B). Accordingly, the expression of TRAP, cathepsin K, and MMP-9, downstream target genes of NFATc1, was also decreased in the presence of capric acid (Figs. 2A and 2B).

Capric acid suppresses RANKL-mediated NF-KB and ERK signaling pathways

We analyzed RANKL signaling pathways to investigate the mechanism by which capric acid inhibits RANKL-induced osteoclastogenesis. As NF-kB activation by RANKL is essential for osteoclast differentiation, we first assessed NF- $\mathrm{kB}$ activation in the presence of capric acid. BMMs were pre-treated with vehicle or capric acid and then stimulated with RANKL. Although RANKL induced profound phosphorylation of $I_{\kappa} B \alpha$ in the vehicle-treated control cells, the addition of capric acid abrogated $I_{\kappa} B \alpha$ phosphorylation in response to RANKL (Fig. 3A). To further confirm its inhibitory effect on NF-kB, we evaluated the nuclear translocation of the p65 NF- $\kappa B$ subunit. As shown in Fig. 3B, capric acid reduced the nuclear translocation of $\mathrm{p} 65$ by RANKL but did not affect the nuclear translocation of phospho-c-Jun. We next examined the impact of capric acid on RANKL-mediated NF-KB transcriptional activity in the RAW264.7 macrophage cell line. RANKL induced NF- $\kappa B$ activity, though the induction of NF- $\kappa B$ promoter activity was significantly suppressed by capric acid in a dose-dependent manner (Fig. 3C).

As the MAPK pathway is also important for osteoclastogenesis, we next analyzed the impact of capric acid on the MAPK signaling cascade following RANKL stimulation. Capric acid significantly inhibited RANKL-induced ERK phosphorylation but did not affect the phosphorylation of JNK and p38 (Fig. 3D). Taken together, these results demonstrate that capric acid inhibits osteoclastogenesis by suppressing the NF-KB and ERK activation induced by RANKL.

\section{Capric acid blocks osteoclast cytoskeletal organization}

Osteoclasts are unique bone-resorbing cells. As osteoclasts mature, the cell cytoskeleton undergoes a dramatic reorganization, an event that involves the formation of an actin ring, a cytoskeletal structure critical for efficient bone resorption in osteoclasts. To investigate whether capric acid affects the organization of the osteoclast cytoskeleton, we first focused on osteoclast morphology. BMMs were cultured in the presence of RANKL and M-CSF for 3 days. The cells were then treated with vehicle or capric acid for $16 \mathrm{~h}$ and stained for TRAP. Although the vehicle-treated control cells formed well-spread, characteristic osteoclasts, the capric acid-treated cells failed to spread and formed small TRAP-positive polykaryons (Figs. 4A and 4B). These results suggest that capric acid regulates osteoclast cytoskeletal organization. To determine whether this is the case, we examined the impact of capric acid on the formation of the actin cytoskeleton and performed an actin ring re-formation assay (Kim et al., 2006). To this end, fully differentiated osteoclasts were generated on bone slices and washed with cold cytokine- 
A
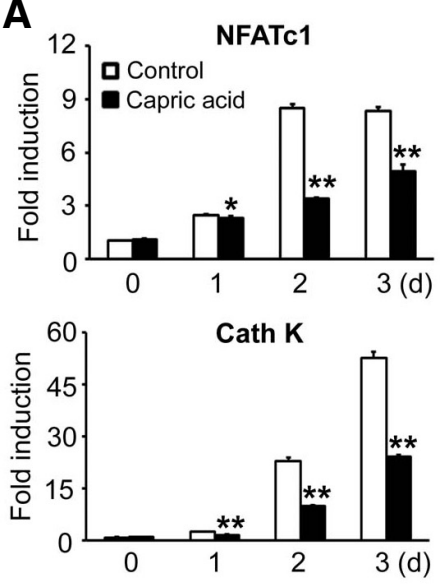

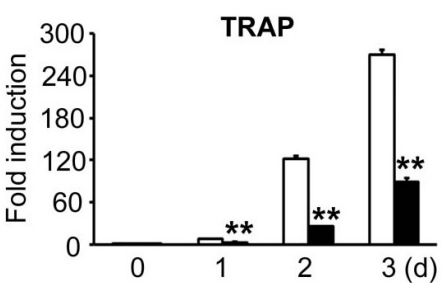

B

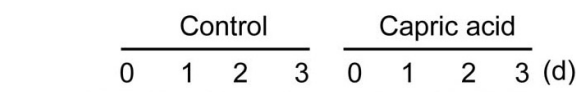

NFATC1

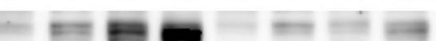

Cath K

$\beta$-actin

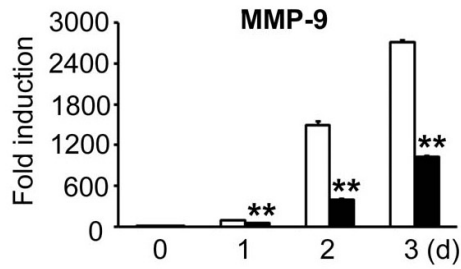

Fig. 2. Effect of capric acid on the expression of osteoclastogenic markers. BMMs were cultured with or without capric acid $(500 \mu M)$ in the presence of M-CSF $(10 \mathrm{ng} / \mathrm{ml})$ and RANKL $(20 \mathrm{ng} / \mathrm{ml})$ for the indicated number of days. Real-time PCR (A) or immunoblotting (B) was performed to assess the expression of the indicated genes. The data presented in (A) are expressed as the means $\pm \mathrm{SD}$. ${ }^{\star} P<0.05$, ${ }^{\star \star} P<0.001$ versus vehicle-treated control (Con).

A

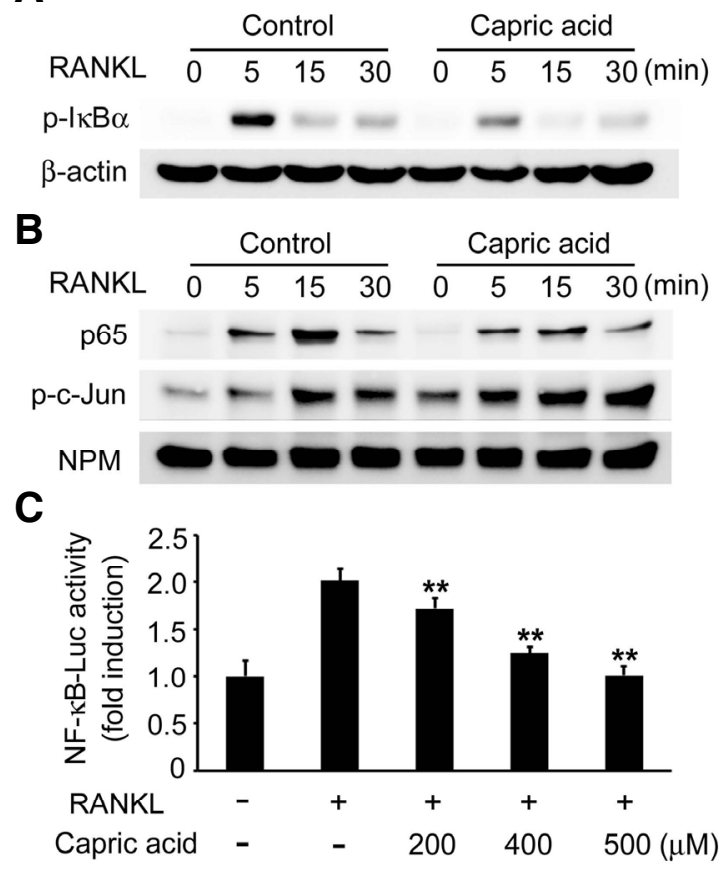

D

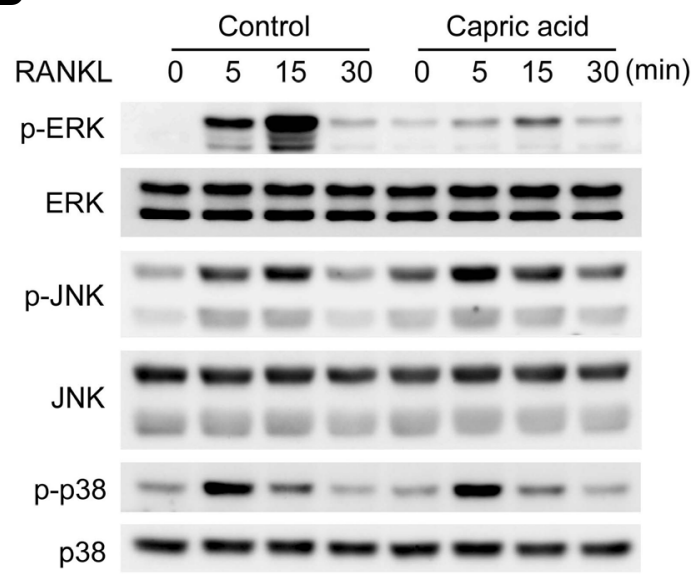

Fig. 3. Effect of capric acid on RANKL-mediated signaling. (A) BMMs were pretreated with capric acid (500 $\mu \mathrm{M})$ or vehicle for $2 \mathrm{~h}$ and then stimulated by the addition of RANKL $(50 \mathrm{ng} / \mathrm{ml})$ for the indicated time. Total cell lysates were immunoblotted with an anti-phospho-lкB $\alpha$ antibody. $\beta$-Actin served as a loading control. (B) The nuclear levels of p65 and phospho-c-Jun in the nuclear extracts of BMMs, as described in (A), were determined by immunoblotting. (C) RAW264.7 cells were transfected with an NF-kB reporter luciferase plasmid. At $24 \mathrm{~h}$ after transfection, the cells were pretreated with the indicated concentration of capric acid and then stimulated with RANKL (200 $\mathrm{ng} / \mathrm{ml})$. After $24 \mathrm{~h}$, the cells were lysed, and the luciferase activity was determined. The data are expressed as the means $\pm S D$. ${ }^{\star *} P<0.001$ versus RANKL alone. (D) Cells were prepared as in (A) and analyzed by immunoblotting with the indicated antibodies. The total ERK, JNK, and p38 levels served as loading controls.

free media to remove the actin rings. The cells were then incubated for $3 \mathrm{~h}$ in the presence of M-CSF or RANKL with or without capric acid. In keeping with its suppressive effect on osteoc- last spreading (Fig. 4A), capric acid blocked the actin rig formation induced by M-CSF or RANKL (Figs. 4C and 4D). Finally, we tested the impact of capric acid on the expression of $\beta 3$ integrin, 
Effect of Capric Acid on Osteoclast Development

Hyun-Ju Kim et al.

A

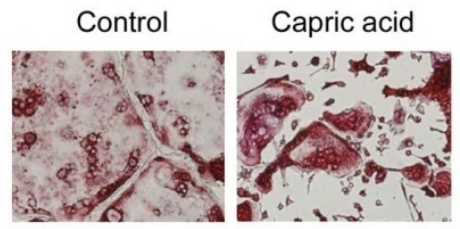

C

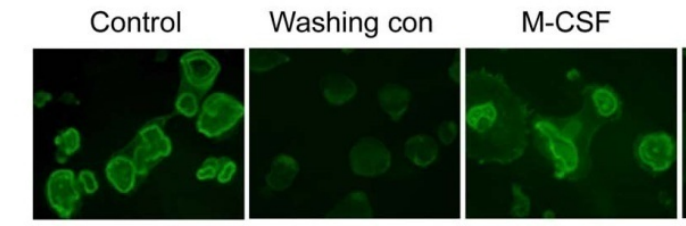

D

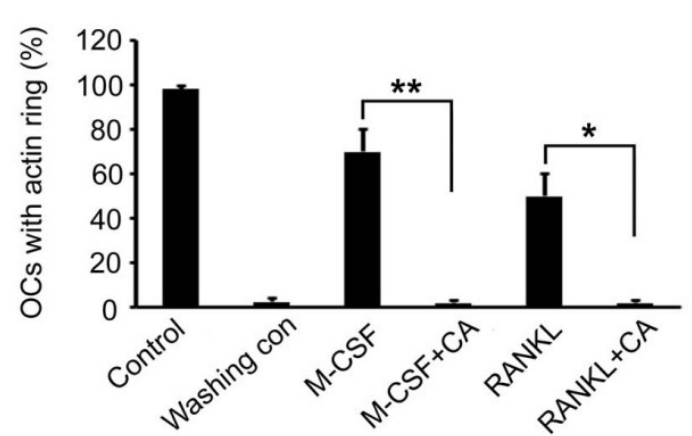

C
B

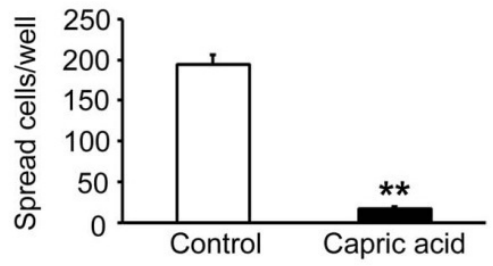

$\mathrm{M}-\mathrm{CSF}+\mathrm{CA}$

RANKL

RANKL + CA
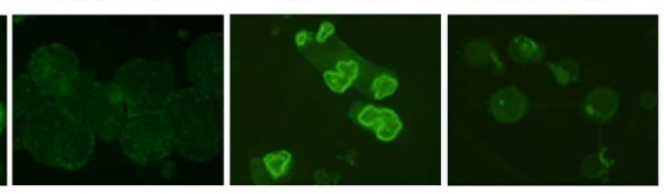

E

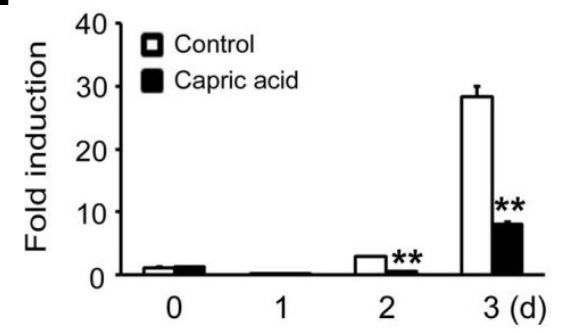

Fig. 4. Effect of capric acid on organization of osteoclast cytoskeleton. (A, B) BMMs were cultured in an osteoclastogenic medium containing $M-$ CSF $(10 \mathrm{ng} / \mathrm{ml})$ and RANKL $(20 \mathrm{ng} / \mathrm{ml})$ for 3 days. The cells were then treated with capric acid or vehicle for $16 \mathrm{~h}$. (A) The cells were fixed and stained for TRAP. (B) The number of spreading TRAP-positive cells was counted. The data are expressed as the means \pm SD. ${ }^{\star \star} P<0.001$. (C, D) Mature osteoclasts cultured on bone slices were washed with cytokine-free cold media (Washing control) to disrupt the actin rings. The cells were then incubated without or with capric acid $(400 \mu \mathrm{M})$ in the presence of M-CSF $(50 \mathrm{ng} / \mathrm{ml})$ or RANKL $(20 \mathrm{ng} / \mathrm{ml})$. (C) After $3 \mathrm{~h}$ of incubation, the cells were fixed and stained with FITC-phalloidin. (D) The percentage of osteoclasts with an actin ring was determined. ${ }^{\star} P<0.05$, ${ }^{\star *} P<$ 0.001 . (E) BMMs were cultured with M-CSF and RANKL for the indicated number of days in the presence or absence of capric acid. $\beta 3$ integrin expression was analyzed by real-time PCR. ${ }^{* \star} P<0.001$ versus vehicle-treated control.

which plays a crucial role in the organization of the osteoclast cytoskeleton. We observed that capric acid significantly attenuated the mRNA levels of $\beta 3$ integrin (Fig. 4E).

\section{Capric acid accelerates apoptosis in mature osteoclasts}

To examine whether capric acid affects osteoclast survival, mature osteoclasts were cultured with or without capric acid for $24 \mathrm{~h}$ and then stained. TRAP staining showed that capric acid reduced the percentage of survival of TRAP-positive multinucleated cells (Figs. 5A and 5B). We next performed an apoptosis assay on these cells using DNA fragmentation ELISA to determine whether the decreased number of osteoclasts observed following capric acid treatment were a direct consequence of cellular apoptosis. As shown in Fig. 5C, capric acid accelerated apoptosis in mature osteoclasts. As osteoclast apoptosis is regulated by a pro-apoptotic Bcl-2 family protein, Bim (Akiyama et al., 2003), we then determined whether capric acid exposure increases Bim expression in osteoclasts. The addition of capric acid significantly increased Bim expression (Fig. 5D). M-CSFinduced ERK activation promotes osteoclast survival by downregulating Bim expression (Akiyama et al., 2003). Therefore, we next examined the impact of capric acid on the M-CSF-induced ERK signaling pathway. Indeed, we observed that capric acid suppressed ERK phosphorylation in response to M-CSF (Fig. 5E).

\section{DISCUSSION}

Excessive bone resorption by osteoclasts plays a critical role in bone-destructive diseases, such as osteoporosis and rheumatoid arthritis. Thus, suppressing osteoclast formation and/or activity may represent a useful therapeutic approach for the treatment of osteoclast-related disorders. In this study, we report that a medium-chain fatty acid, capric acid, suppresses osteoclast differentiation, function, and survival. Capric acid inhibits both the differentiation of precursor cells to TRAP-positive multinuclear osteoclasts and the cytoskeletal organization and survival of mature osteoclasts.

The activation of NF- $\mathrm{KB}$ by RANKL is a critical event for osteoclast differentiation. The essential role of NF-KB in osteoclastogenesis and bone homeostasis has been described by observations from genetic studies. Deletion of the NF-kB subunits $\mathrm{p} 50$ and p52 causes severe osteopetrosis in mice due to defective osteoclast differentiation (Franzoso et al., 1997; lotsova et al., 1997). In the present study, we found that capric acid significantly inhibited the RANKL-mediated activation of NF- $\mathrm{KB}$ signaling cascades, as demonstrated by the suppression of $I_{\kappa} B \alpha$ phos- 
A

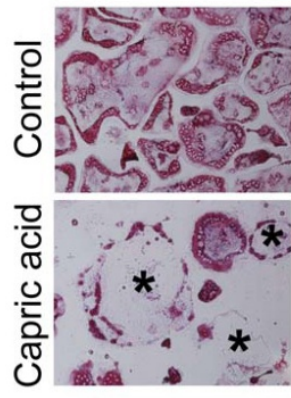

B

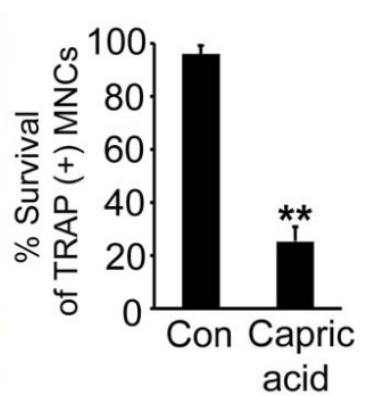

E

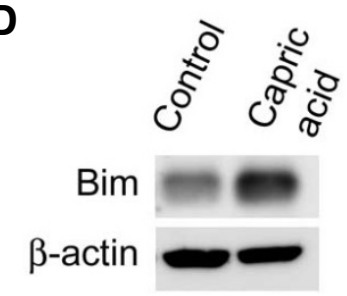

C

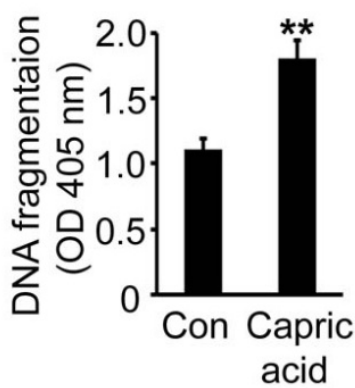

Fig. 5. Effect of capric acid on survival of mature osteoclast. Mature osteoclasts were treated with capric acid $(500 \mu \mathrm{M})$ or vehicle for $24 \mathrm{~h}$. (A) The cells were fixed and stained for TRAP. (B) Osteoclast survival was measured as the percentage of TRAP-positive MNCs. (C) The rate of osteoclast apoptosis was measured by ELISA. The data presented in (B) and (C) are expressed as the means \pm SD. ${ }^{\star *} P<0.001$. (D) The Bim content was determined by immunoblotting. $\beta$-Actin served as a loading control. (E) Cells were treated with capric acid $(500 \mu \mathrm{M})$ or vehicle and then exposed to M-CSF (50 $\mathrm{ng} / \mathrm{ml})$ for $5 \mathrm{~min}$. Total cell lysates were immunoblotted with an anti-phospho-ERK antibody. Total ERK served as a loading control. phorylation, p65 nuclear translocation, and NF- $\mathrm{KB}$ transcriptional activity. In addition to NF-кB pathway, three well-known MAPKs (ERK, JNK, and p38) are also activated by RANKL stimulation and play important roles in osteoclast differentiation. Previous studies by our and other groups have demonstrated that the inhibition of ERK activation suppresses osteoclast formation ( $\mathrm{He}$ et al., 2012; Kim et al., 2007; 2014a). In the present study, capric acid specifically blocked the phosphorylation of ERK by RANKL, whereas it did not affect the activation of JNK and p38.

NFATc1 is a master regulator in osteoclastogenesis, and its induction is a critical step that determines the cell fate of osteoclasts (Asagiri and Takayanagi, 2007). It has been reported that NFATc1 is a key target gene of NF- $\mathrm{KB}$ at the early phase of osteoclastogenesis. Asagiri et al. (2005) demonstrated that NF$\kappa B$ subunits $\mathrm{p} 50$ and $\mathrm{p} 65$ are recruited to the NFATc1 promoter after RANKL stimulation and also showed that the overexpression of NF- $\mathrm{kB}$ activated NFATc1 promoter activity in a luciferase reporter assay (Asagiri et al., 2005). We observed that the addition of capric acid suppressed NF- $\kappa$ B activation and blocked NFATc1 mRNA and protein expression. These results indicate that capric acid inhibits NF- $\mathrm{KB}$ and subsequently reduces NFATc1 gene expression during RANKL-induced osteoclastogenesis. Thus, we suggest that an impaired NF-kB signal may contribute to the suppressive effect of capric acid on NFATc1 expression.

Mature osteoclasts attach to bone matrix and undergo characteristic morphological changes that involve the formation of an actin ring or sealing zone. These polarizing events are pivotal for osteoclastic bone resorption and require the activation of $\alpha \mathrm{v} \beta 3$ integrin, a key modulator of cytoskeletal organization in mature osteoclasts (Faccio et al., 2003; McHugh et al., 2000). Osteoclasts from $\beta 3$ integrin-deficient mice were unable to resorb bone as a result of defective cell spreading and cytoskeletal organization, leading to increased bone density (McHugh et al., 2000). In the present study, we observed that capric acid-treated osteoclasts could not spread, resulting in small and abnormal polykaryons. The morphological similarity between $\beta 3$ integrin-lacking osteoclasts and capric acid-treated cells raised the possibility that capric acid might affect the cytoskeletal organization in mature osteoclasts. In fact, we found that capric acid blocked the actin ring re-formation induced by two cytokines, RANKL and MCSF, establishing that capric acid regulates the osteoclast cytoskeleton. These results are consistent with our previous findings that showed that defective cell spreading is accompanied by failure in osteoclast cytoskeleton organization (Hong et al., 2011; Kim et al., 2006; 2014a; 2014b). In addition, capric acid attenuated the expression of the $\beta 3$ integrin subunit. Therefore, the suppressive effect of capric acid on $\beta 3$ integrin expression likely contributes to the related cytoskeleton disorganization.

Osteoclastic bone resorption is also governed by the rate of osteoclast apoptosis. Bim is a pro-apoptotic BH3-only Bcl-2 family member, and its expression level is critical for controlling osteoclast apoptosis. Osteoclasts from Bim-deficient mice showed enhanced osteoclast survival (Akiyama et al., 2003). In this study, we observed that capric acid accelerated apoptosis in mature osteoclasts by the induction of Bim expression. These results suggest that increased expression of the pro-apoptotic protein Bim could be associated with capric acid-induced apoptosis in osteoclastic cells.

In this study, we demonstrated that a medium-chain fatty acid, capric acid, inhibits RANKL-induced osteoclast differentiation. These inhibitory effects occur through the suppression of NF-kB and ERK activation and the attenuation of NFATc1 induction. In agreement with our finding, a previous study reported that capric acid inhibits lipopolysaccharide-induced osteoclastogenesis through the suppression of nitric oxide production and signal transducer and activator of transcription 3 activation (Park et al., 2011). Thus, data from both studies clearly indicate that capric acid has anti-osteoclastogenic property. Importantly, our study further shows the novel inhibitory action of capric acid on osteoclast cytoskeleton and survival. Therefore, we suggest that capric acid may become a novel dietary therapeutic agent against osteoclast-related diseases.

\section{ACKNOWLEDGMENTS}

This research was supported by the Basic Science Research 
Program of the National Research Foundation of Korea (NRF) funded by the Ministry of Education, Science, and Technology (2013R1A1A2A10005515).

\section{REFERENCES}

Akiyama, T., Bouillet, P., Miyazaki, T., Kadono, Y., Chikuda, H. Chung, U.I., Fukuda, A., Hikita, A., Seto, H., Okada, T., et al. (2003). Regulation of osteoclast apoptosis by ubiquitylation of proapoptotic BH3-only Bcl-2 family member Bim. EMBO J. 22, 6653-6664.

Asagiri, M., and Takayanagi, H. (2007). The molecular understanding of osteoclast differentiation. Bone 40, 251-264.

Asagiri, M., Sato, K., Usami, T., Ochi, S., Nishina, H., Yoshida, H., Morita, I., Wagner, E.F., Mak, T.W., Serfling, E., et al. (2005). Autoamplification of NFATc1 expression determines its essential role in bone homeostasis. J. Exp. Med. 202, 1261-1269.

Boyle, W.J., Simonet, W.S., and Lacey, D.L. (2003). Osteoclast differentiation and activation. Nature 423, 337-342.

Cornish, J., MacGibbon, A., Lin, J.M., Watson, M., Callon, K.E., Tong, P.C., Dunford, J.E., van der Does, Y., Williams, G.A., Grey, A.B., et al. (2008). Modulation of osteoclastogenesis by fatty acids. Endocrinology 149, 5688-5695.

Faccio, R., Zou, W., Colaianni, G., Teitelbaum, S.L., and Ross, F.P. (2003). High dose M-CSF partially rescues the Dap12-/- osteoclast phenotype. J. Cell. Biochem. 90, 871-883.

Franzoso, G., Carlson, L., Xing, L., Poljak, L., Shores, E.W., Brown, K.D., Leonardi, A., Tran, T., Boyce, B.F., and Siebenlist, U. (1997). Requirement for NF-kappaB in osteoclast and B-cell development. Genes. Dev. 11, 3482-3496.

He, L., Lee, J., Jang, J.H., Lee, S.H., Nan, M.H., Oh, B.C., Lee, S.G. Kim, H.H., Soung, N.K., Ahn, J.S., et al. (2012). Ginsenoside Rh2 inhibits osteoclastogenesis through down-regulation of NFkappaB, NFATc1 and c-Fos. Bone 50, 1207-1213.

Hong, J.M., Teitelbaum, S.L., Kim, T.H., Ross, F.P., Kim, S.Y., and Kim, H.J. (2011). Calpain-6, a target molecule of glucocorticoids, regulates osteoclastic bone resorption via cytoskeletal organization and microtubule acetylation. J. Bone Miner. Res. 26, 657-665.

lotsova, V., Caamano, J., Loy, J., Yang, Y., Lewin, A., and Bravo, R. (1997). Osteopetrosis in mice lacking NF-kappaB1 and NFkappaB2. Nat. Med. 3, 1285-1289.

Kim, H.J., Zhao, H., Kitaura, H., Bhattacharyya, S., Brewer, J.A Muglia, L.J., Ross, F.P., and Teitelbaum, S.L. (2006). Glucocorticoids suppress bone formation via the osteoclast. J. Clin. Invest.
116, 2152-2160.

Kim, H.J., Lee, Y., Chang, E.J., Kim, H.M., Hong, S.P., Lee, Z.H., Ryu, J., and Kim, H.H. (2007). Suppression of osteoclastogenesis by N,N-dimethyl-D-erythro-sphingosine: a sphingosine kinase inhibition-independent action. Mol. Pharmacol. 72, 418-428.

Kim, H.J., Hong, J.M., Yoon, K.A., Kim, N., Cho, D.W., Choi, J.Y., Lee, I.K., and Kim, S.Y. (2012). Early growth response 2 negatively modulates osteoclast differentiation through upregulation of Id helix-loop-helix proteins. Bone 51, 643-650.

Kim, H.J., Hong, J.M., Yoon, H.J., Kwon, B.M., Choi, J.Y., Lee, I.K., and Kim, S.Y. (2014a). Inhibitory effects of obovatol on osteoclast differentiation and bone resorption. Eur. J. Pharmacol. 723, 473480.

Kim, H.J., Yoon, H.J., Choi, J.Y., Lee, I.K., and Kim, S.Y. (2014b). The tyrosine kinase inhibitor GNF-2 suppresses osteoclast formation and activity. J. Leukoc. Biol. 95, 337-345.

Martin, T.J. (2014). Bone biology and anabolic therapies for bone: current status and future prospects. J. Bone Metabol. 21, 8-20.

McHugh, K.P.. Hodivala-Dilke, K., Zheng, M.H., Namba, N., Lam, J., Novack, D., Feng, X., Ross, F.P., Hynes, R.O., and Teitelbaum, S.L. (2000). Mice lacking beta3 integrins are osteosclerotic because of dysfunctional osteoclasts. J. Clin. Invest. 105, 433-440.

Nakashima, T., and Takayanagi, H. (2011). New regulation mechanisms of osteoclast differentiation. Ann. N Y Acad. Sci. 1240, E1318.

Novack, D.V., and Teitelbaum, S.L. (2008). The osteoclast: friend or foe? Ann. Rev. Pathol. 3, 457-484.

Park, E.J., Kim, S.A., Choi, Y.M., Kwon, H.K., Shim, W., Lee, G., and Choi, S. (2011). Capric acid inhibits NO production and STAT3 activation during LPS-induced osteoclastogenesis. PLoS One 6, e27739.

Rahman, M.M., Bhattacharya, A., and Fernandes, G. (2008). Docosahexaenoic acid is more potent inhibitor of osteoclast differentiation in RAW 264.7 cells than eicosapentaenoic acid. J. Cell. Physiol. 214, 201-209

Sun, D., Krishnan, A., Zaman, K., Lawrence, R., Bhattacharya, A., and Fernandes, G. (2003). Dietary n-3 fatty acids decrease osteoclastogenesis and loss of bone mass in ovariectomized mice. J. Bone Miner. Res. 18, 1206-1216.

Teitelbaum, S.L. (2007). Osteoclasts: what do they do and how do they do it? Am. J. Pathol. 170, 427-435.

Teitelbaum, S.L., and Ross, F.P. (2003). Genetic regulation of osteoclast development and function. Nat. Rev. Genet. 4, 638-649.

Vaananen, H.K., Zhao, H., Mulari, M., and Halleen, J.M. (2000). The cell biology of osteoclast function. J. Cell Sci. 113 ( Pt 3), 377-381. 